\title{
LA EXPERIENCIA DEMOCRÁTICA EN EL LABERINTO DE LAS CULTURAS POLÍTICAS
} THE DEMOCRATIC EXPERIENCE IN THE LABYRINTH OF POLITICAL CULTURES

Bernardo Carrizo de la Facultad de Humanidades y Ciencias (Universidad Nacional del Litoral. 


\section{RESUMEN}

El artículo presenta los principales lineamientos de un proyecto de investigación que atiende al entramado de culturas políticas que constituyen a la democracia en sus múltiples rostros. La investigación se propone una articulación entre lo nacional, subnacional y regional en torno a la democracia argentina como experiencia histórica, durante el siglo XX y principios del XXI. Estas coordenadas témporo-espaciales se sostienen en la hipótesis según la cual pueden reconocerse diferentes espacios de producción de lo político y la política de tal modo que su estudio contribuye a iluminar y matizar aspectos del proceso histórico difíciles de recuperar en una visión estrictamente centrada en la perspectiva nacional. El proyecto construye su objeto buscando recuperar la articulación entre prácticas, discursos e ideas en tanto que componentes de las culturas políticas que pueden reconocerse en el período. A través del proyecto se aspira a consolidar un enriquecedor diálogo entre disciplinas, pues los fenómenos políticos son dinámicos y los límites de lo político son flexibles, por lo que se apelará a una construcción metodológica compleja e interdisciplinar de observación para poder adaptarse a los altos niveles de incertidumbre, contingencia y riesgo que el objeto convoca.

\section{PALABRAS CLAVE}

$>$ culturas políticas

$>$ experiencia democrática

$>$ escalas de análisis 


\section{ABSTRACT}

This article presents the main guidelines of a research project that addresses the network of political cultures that constitute democracy in its many faces. The research proposes an articulation between the national, subnational and regional aspects of Argentine democracy as a historical experience, during the 20th century and the beginning of the 21st century. These temporal-spatial coordinates are supported by the hypothesis according to which different spaces of politics production can be recognized in such a way that their study contributes to illuminate and clarify aspects of the historical process that are difficult to recover in a vision strictly centered on the national perspective. The project builds its object by seeking to recover the articulation between practices, discourses and ideas as components of political cultures that can be recognized in the period. The project aims to consolidate an enriching dialogue between disciplines, since political phenomena are dynamic and the limits of the political are flexible, so it will appeal to a complex and interdisciplinary methodological construction of observation in order to adapt to the high levels of uncertainty, contingency and risk that the object summons.

\section{KEYWORDS}

$>$ political cultures

$>$ democratic experience

$>$ analysis scales 


\section{INTRODUCCIÓN}

El proyecto de investigación «Culturas políticas en escalas. La experiencia democrática entre lo nacional, subnacional y regional (siglos $\mathrm{XX}-\mathrm{XXI}$ )»1 se propone continuar, con reformulaciones de diverso tipo, una línea de investigación centrada en las culturas políticas iniciada en uno similar presentado en la programación CAI+D 2016, y que da cuenta de una forma de indagación de los objetos políticos al estilo de una caja de herramientas, antes que como teoría general.

La enriquecedora variación de escalas de análisis - lo nacional, lo subnacional y lo regional, que a su vez conforman diversos territorios de producción de lo político- permite la comprensión de fenómenos que la perspectiva nacional coloca en un plano de inicial homogeneidad. Cada escala ofrece componentes de veracidad, susceptibles de entrar en conflicto con los aportados por las demás. Tanto las coordenadas temporales como las espaciales se sostienen en la hipótesis según la cual pueden reconocerse diferentes áreas de producción de lo político y la política.

A partir de las líneas de investigación que el proyecto cobija se aspira, por un lado, a que los resultados contribuyan a nuevos análisis en perspectiva multiescalar. Por otro lado, se pretende formular un marco referencial de interpretación que pueda dar cuenta de una reconstrucción histórica y de las reconfiguraciones permanentes que atañen al campo de poder y su articulación con la experiencia democrática.

\footnotetext{
${ }^{1}$ El proyecto CAI+D fue aprobado por la Secretaría de Ciencia, Arte y Tecnología de la UNL en la convocatoria 2020. El grupo responsable está integrado por Bernardo Carrizo (director), Esteban Kaipl (co-director), Juan Cruz Giménez, Marcelino Maina y Cecilia Rambaudo. El grupo colaborador, por Celina Albornoz, Agustín Arnaudo, Manuela Giménez Bautista, Katia Ingerman, Corina Marenoni, Mariano Rinaldi, Camillo Robertini, Javier Rodrigo y Leila Romero.
} 


\section{ESTADO DE LA CUESTIÓN Y MARCO TEÓRICO DE REFERENCIA}

En las últimas décadas, los diálogos entre las ciencias sociales han enriquecido el análisis de las tramas que componen el campo de poder. Numerosos trabajos hicieron posible la «recuperación de la centralidad de la esfera política, o más genéricamente, del ámbito de 〈lo político en la historia» (Barriera, 2002:185). A través de esa articulación entre diversas dimensiones y herramientas provenientes de diferentes disciplinas, se puede dar cuenta de un intercambio que posibilita enriquecer una observación dinámica sobre la reconfiguración contingente de lo político en el mundo contemporáneo. Por ejemplo, los modos de hacer política, las percepciones de los actores sobre lo político y el sentido de la política.

Una serie de producciones e investigaciones han expuesto un entramado de temas -escenarios parlamentarios y electorales, lucha armada y violencia política, historia y memoria, actores sociales y políticos, intelectuales y política, organizaciones y bloques de poder, agencias estatales y burocracia, elites y dirigencias, por citar algunos - en donde la política y lo político atraviesan a la democracia como experiencia histórica en el caso argentino —ella misma sistemáticamente interpelada como fenómeno social en cotejo con lo que idealmente supone-, y donde lo nacional, subnacional y regional explicitan distintas escalas de observación (Revel, 2011; 2015).

En esta clave, la literatura académica abordó problemáticas instaladas en diversos espacios y marcos temporales que han puesto en evidencia una serie de problemáticas en las que la política - las instituciones y normas vinculadas al poder y su ejercicio - y lo político - aquello que anima a los sujetos a desplegar sus prácticas y que guarda relación con los conflictos por la hegemonía (Rosanvallon, 2003a) - atraviesan el campo de poder. Nuestro supuesto es que uno de los aspectos donde puede detenerse el punto de observación para analizar la experiencia democrática radica en las culturas políticas que participan en su invención, y reinvención permanente a partir de la experiencia (Guénard, 2019).

La cultura política - fructífera herramienta conceptual en clave heurística-es un componente de la renovación que experimentó la historia política a partir de aportes generados en las últimas décadas (Sirinelli, 1993; Morán, 1999; Berstein, 1999, 2013; Rosanvallon, 2003a; de Diego Romero, 2006; Angenot, 2010; Pérez Ledesma y Sierra, 2010; Cabrera y Pro, 2014; Tabanera y Bonaudo, 2016). 
El concepto proporciona un abanico de matices que puede darnos las trazas para reconocer la complejidad de los comportamientos humanos e indagar la dinámica de actores - en términos individuales y colectivos - e instituciones en relación a los procesos políticos y sociales.

El análisis de los discursos y las prácticas simbólicas junto a los valores, representaciones y objetivos específicos son los elementos que, en principio, conforman las diversas culturas políticas reconocibles en un recorrido procesual. Desde esta perspectiva, profundizar el análisis de las culturas políticas implica comprender los motivos que conducen a los actores a asumir ciertos comportamientos políticos, a optar por ciertos principios de legitimidad o por un sentido del orden (Berstein, 1999).

Solo como forma de exponer algunos de los ingredientes que dan cuenta de estos procedimientos y sentidos que son constitutivos de las culturas políticas, es posible mencionar: una visión del mundo, una lectura común y normativa del pasado que pone en relevancia ciertos hechos como gesta o hito, una definición sobre el tipo de organización política deseable, un discurso significativo (sobre temas como nación, religión, historia, memoria) cuyos componentes (palabras clave, consignas, figuras idealizadas) dan cuenta - junto a ciertos rituales, símbolos, publicaciones y sociabilidad- de la presencia de conflictos y tensiones, como así también la aspiración a cristalizarse en alguna forma de ocupación del espacio público (movilizaciones, concentraciones, actos, homenajes). Como puede observarse, «las culturas moldean gradualmente, tomando elementos del pasado y dándoles nuevas interpretaciones, apropiaciones, reformulaciones [...] todo ello con un ritmo más o menos lento, pero sin cortes» (Cabrera y Pro, 2014:23).

Acordamos con el historiador francés Serge Berstein sobre «el carácter plural de las culturas políticas en un momento dado de la historia y en un país determinado» (1999:390) ya que puede brindar puntos de contacto —al estilo de un mosaico - sumamente estimulante para el análisis de la historia argentina en diversas escalas. La pluralidad de culturas políticas obliga, además, a traer nuevamente al ruedo un dispositivo analítico sumamente relevante para la historiografía como lo es el vínculo continuidad / ruptura. La configuración, interacción, transversalidad y transnacionalidad de las culturas políticas (Cabrera y Pro, 2014) obligan a un diálogo interesante con ellas para que puedan ofrecer nuevos horizontes en la comprensión de los fenómenos políticos. 
A partir de las culturas políticas, el recorrido por la experiencia democrática en Argentina no resulta una tarea sencilla y menos aún acabada. Diversas culturas pueden reconocerse en un período más que secular: liberal, republicana, liberal conservadora, antiliberal reaccionaria, nacionalista(s), libertaria, socialista, autoritaria(s), populista, democrática. En realidad, aquéllas son tipos ideales vinculados con tradiciones de pensamiento teórico-político que la investigación histórica posibilita reconocer en permanente puja por la hegemonía en ciertas coyunturas. Asimismo, en tanto orientadores de sentido del conocimiento por parte del observador de lo social, construyen identidades políticas («es un autoritario», "soy liberal», "milito en el socialismo») y participan en la construcción de regímenes políticos (democracias, dictaduras) como también de organizaciones políticas, aunque no pueda reconocerse indefectiblemente una relación de causa-efecto.

En una somera presentación de las culturas políticas para el caso argentino, podemos señalar que en las primeras décadas del siglo XX la cultura política liberal recorrió tanto su esplendor como su ocaso y, a su vez, propició una serie de reflexiones sobre la democracia de masas, la relación entre democracia, organización y burocracia, y la consideración de alternativas autoritarias frente a la experiencia democrática. En una mirada más atenta, otras culturas políticas ganaban densidad a medida que avanzaba el siglo sin que necesariamente impusieran la desaparición de aquélla. Podrían mencionarse las diferentes vertientes del nacionalismo - y su relación con la tradición católica- que aspira al orden y a las soluciones autoritarias; las huellas que deja el populismo al plantear un horizonte societal inclusivo con una lectura sustancial de la democracia, y las identidades combativas forjadas al calor de la crisis de legitimidad de la democracia, como camino de resolución de los conflictos pero también como construcción de un horizonte que se propone lo más justo posible. Y, por último, las apuestas a la construcción de una experiencia democrática que abreva en esas culturas políticas y otorgan complejidades y desafíos en el recorrido político más reciente. 


\section{OBJETIVOS}

\section{GENERAL}

$>$ Analizar el funcionamiento del sistema político (competencia electoral, acción legislativa, discursos, relación gobierno/oposición) y las transformaciones del Estado, en escalas nacional, subnacional y regional, en tanto aspectos que dan cuenta de la coexistencia de determinadas culturas políticas en períodos específicos y la articulación de elementos conceptuales propios de las tradiciones de teoría política diferenciadas (liberalismo, democracia y republicanismo).

\section{ESPECÍ́FICOS}

$>$ Analizar los discursos políticos como prácticas, cuyo entramado de relaciones moldean las intervenciones en la esfera pública.

$>$ Rastrear la presencia de elementos propios de las tradiciones de pensamiento político (democracia, liberalismo, republicanismo) en los discursos.

$>$ Explorar los temas, debates y argumentaciones más sobresalientes de la agenda parlamentaria, destacando el posicionamiento de los diferentes bloques partidarios.

$>$ Identificar las principales políticas impulsadas por el Ejecutivo, atendiendo a su contenido y fundamentaciones.

$>$ Detectar los cambios en las estructuras estatales, poniendo especial atención en las ideas e influencias que fundamentan las innovaciones, así como en los perfiles de funcionarios y técnicos.

$>$ Indagar acerca de la emergencia de actores con influencia política más allá de los centrados en el Estado.

$>$ Distinguir líneas de continuidad o quiebre en el proceso de conformación de las políticas estatales. 


\section{ESTRATEGIA METODOLÓGICA}

La investigación aspira a consolidar un enriquecedor diálogo entre disciplinas, pues los fenómenos políticos son dinámicos y los límites de lo político son flexibles, por lo que apelaremos a una construcción metodológica compleja e interdisciplinar de observación para poder adaptarse a los altos niveles de contingencia y de riesgo (Luhmann, 1992; Beck, 1998; Habermas, 1999; Mouffe, 2000; Rosanvallon, 2003b, 2007,2020; Quiroga, 2016).

La investigación es diacrónica y se encuadra en una metodología cualitativa. El análisis de la producción académica junto a los documentos será el procedimiento más frecuente para el acceso a datos que permitan visibilizar el entramado de culturas políticas. Entre los documentos pueden mencionarse: leyes y decretos, diarios de sesiones legislativas, mensajes de apertura de sesiones parlamentarias, prensa y revistas de publicación regular. En función de los objetivos de la investigación y de la formulación de hipótesis se avanzará en un análisis que resulte del diálogo y la puesta en tensión entre categorías e indagación empírica.

Nos detendremos en el análisis del sistema político y las prácticas políticas, poniendo el foco en las organizaciones partidarias $y$, especialmente, en los escenarios en los que estas despliegan su accionar principal: el electoral y el parlamentario. En esos escenarios exclusivos de las organizaciones partidarias, se atenderá el comportamiento electoral, las reglas que organizan las competencias, la composición de las cámaras legislativas, los principales debates, argumentaciones y discursos con el fin de reconstruir la dinámica legislativa. Se procurará inferir la relación poder legislativo / partido de gobierno / poder ejecutivo / partidos de oposición.

La investigación también se propone hacer inteligible el proceso de construcción de las políticas estatales, atendiendo a los contenidos, fundamentos y argumentaciones que se esgrimen. Se atenderá al efecto que ejerce la estructura institucional en las políticas propuestas, y a su vez el impacto de las políticas en esas estructuras. 


\section{BIBLIOGRAFÍA}

Angenot, M. (2010). El discurso social. Los límites históricos de lo pensable y lo decible. Buenos Aires: Siglo XXI.

Barriera, D. (2002). Por el camino de la historia política: hacia una historia política configuracional. Secuencia. Revista de historia y ciencias sociales, 53, 163-196. Disponible en http://secuencia.mora.edu.mx/index.php/Secuencia/article/view/779/690

Beck, U. (1998). Sociedad del riesgo, Hacia una nueva modernidad. Barcelona: Paidós. Berstein, S. (1999). La cultura política. En Rioux, J.P. y Sirinelli, J.F. (dir.). Para una historia cultural (389-405). México: Taurus.

Berstein, S. (2013). Los regímenes políticos del siglo XX. Barcelona: Ariel.

Cabrera, M.A. y Pro, J. (2014). La creación de las culturas políticas modernas (18081833) [Historia de las culturas políticas en España y América Latina, vol. I]. Zaragoza: Marcial Pons Historia/Prensas de la Universidad de Zaragoza.

de Diego Romero, J. (2006). El concepto de «cultura política» en ciencia política y sus implicaciones para la historia, Ayer. Revista de Historia Contemporánea, 61. Disponible en http://www.ahistcon.org/PDF/numeros/ayer61_RepresentacionPoliticaEspanaLiberal_Sierra_Zurita_Pena.pdf

Guénard, F. (2019). La igualdad política y los límites de la democracia procesal. Filosófico, 46 (1), 29-44. Disponible en https://www.erudit.org/en/journals/philoso/1900-v1-n1-philoso04752/1062011ar.pdf

Habermas, J. (1999). La inclusión del otro. Estudios de Teoría Política. Barcelona: Paidós.

Morán, M.L. (1999). Los estudios de cultura política en España. Reis, 97-129. Disponible en http://www.reis.cis.es/REIS/PDF/REIS_085_081208156724438.pdf

Mouffe, Ch. (2000). La paradoja democrática. Barcelona: Gedisa.

Pérez Ledesma, M. y Sierra, M. (eds.) (2010). Culturas políticas: teoría e historia. Zaragoza: Institución «Fernando el Católico» (CSIC).

Quiroga, H. (2016). La democracia que no es. Política y sociedad en la Argentina (1983-2016). Buenos Aires: Edhasa.

Revel, J. (2011). Micro versus Macro: escalas de observación y discontinuidad en la historia. Tiempo histórico: revista de la Escuela de Historia, №2, pp. 15-26. 
Revel, J. (ed.) (2015). Juegos de escalas: experiencias de microanálisis. San Martín: UNSAM Edita.

Rosanvallon, P. (2003a). Por una historia conceptual de lo político. Buenos Aires: FCE. Rosanvallon, P. (2003b). La sociedad de los iguales. Buenos Aires: Manantial.

Rosanvallon, P. (2007). La contrademocracia. Buenos Aires: Manantial.

Rosanvallon, P. (2010). La legitimidad democrática. Barcelona: Paidós.

Sirinelli, J-F. (1993). El retorno de lo político, Historia contemporánea, № 9, 25-35.

Disponible en https://addi.ehu.es/bitstream/handle/10810/37712/19594-73959-1-PB. pdf? sequence $=1$

Tabanera, N. y Bonaudo, M. (2016). América Latina. De la independencia a la crisis del liberalismo (1810-1930) [Historia de las culturas políticas en España y América Latina, vol. V]. Zaragoza: Marcial Pons Historia/Prensas de la Universidad de Zaragoza. 